\title{
Neuraminidase Inhibition Primes Short-Term Depression and Suppresses Long-Term Potentiation of Synaptic Transmission in the Rat Hippocampus
}

\author{
Alina Savotchenko, ${ }^{1}$ Arthur Romanov, ${ }^{1}$ Dmytro Isaev, ${ }^{1,2}$ Oleksandr Maximyuk, ${ }^{1}$ \\ Vadym Sydorenko, ${ }^{1}$ Gregory L. Holmes, ${ }^{2}$ and Elena Isaeva ${ }^{1,2}$ \\ ${ }^{1}$ Department of Cellular Membranology, Bogomoletz Institute of Physiology, Bogomoletz Street 4, Kiev 01024, Ukraine \\ ${ }^{2}$ Department of Neurological Sciences, University of Vermont, 149 Beaumont Avenue, Burlington, VT 05405, USA
}

Correspondence should be addressed to Elena Isaeva; olena.isaeva@gmail.com

Received 22 December 2014; Accepted 16 January 2015

Academic Editor: Michael G. Stewart

Copyright ( $) 2015$ Alina Savotchenko et al. This is an open access article distributed under the Creative Commons Attribution License, which permits unrestricted use, distribution, and reproduction in any medium, provided the original work is properly cited.

\begin{abstract}
Neuraminidase (NEU) is a key enzyme that cleaves negatively charged sialic acid residues from membrane proteins and lipids. Clinical and basic science studies have shown that an imbalance in NEU metabolism or changes in NEU activity due to various pathological conditions parallel with behavior and cognitive impairment. It has been suggested that the decreases of NEU activity could cause serious neurological consequences. However, there is a lack of direct evidences that modulation of endogenous NEU activity can impair neuronal function. Using combined rat entorhinal cortex/hippocampal slices and a specific inhibitor of NEU, 2-deoxy-2,3-dehydro-N-acetylneuraminic acid (NADNA), we examined the effect of downregulation of NEU activity on different forms of synaptic plasticity in the hippocampal CA3-to-CA1 network. We show that NEU inhibition results in a significant decrease in long-term potentiation (LTP) and an increase in short-term depression. Synaptic depotentiation restores LTP in NADNApretreated slices to the control level. These data suggest that short-term NEU inhibition produces the LTP-like effect on neuronal network, which results in damping of further LTP induction. Our findings demonstrate that downregulation of NEU activity could have a major impact on synaptic plasticity and provide a new insight into the cellular mechanism underlying behavioral and cognitive impairment associated with abnormal metabolism of NEU.
\end{abstract}

\section{Introduction}

Long chains of negatively charged sialic acid occupy a prominent position on cellular membrane proteins in complex carbohydrates, which are major constituents of membrane proteins and lipids and are involved in manifold cell signaling events [1]. In the central nervous system, sialic acids play an important role in many processes such as neurogenesis, cell differentiation, migration, axon sprouting, synaptogenesis, plasticity, and neuronal excitability $[2,3]$. Involvement of polysialic acid (PSA), a homopolymer of sialic acid, in a wide range of neuronal functions, related to the ability of PSA to modulate attracting and repulsing molecule-molecule interactions and membrane surface charge density due to their negative charge, bulky size, and location on the outer surface of the membrane $[4,5]$.
The physiological role of sialic acid comes from studies using neuraminidase (NEU) as an enzyme, which hydrolyzes terminal sialic acid residues from cellular glycoconjugates. In most studies, NEU is applied extracellularly to decrease cell sialylation [2, 6-9]. Removal of sialic acid by NEU affects neurogenesis, synaptogenesis, synaptic plasticity, neuronal excitation, and spatial learning and causes behavioral abnormalities [2, 6, 10-12]. Changes of endogenous NEU activity, as a physiological regulator of the level of sialic acid, could also alter neuronal function. Clinical observations indicate that an imbalance in the metabolism of NEU has a significant influence on the function of neuronal systems. Indeed, mental retardation and seizures are common clinical features of inherited disorders of defective or deficient NEU activity $[13,14]$. Various pathological conditions such as chronic stress, seizure activity, and chronic ethanol treatment 
induce changes in NEU activity in different regions of the brain [15-17]. These changes in NEU activity have been suggested to be responsible for physiological and neurological impairment in the brain, presumably due to the effect of NEU on glycosylation [18]. However, there is a lack of direct experimental studies showing that alteration of endogenous NEU activity could affect neuronal function.

Previous in vitro and in vivo studies show that decreases of NEU activity by ganglioside sialidase inhibitors induce synaptogenesis and affect neuronal excitation, which is accompanied by the accumulation of PSA in rat hippocampus $[2,19,20]$. As the deficit of NEU activity is associated with behavior and mental impairment and alterations of the level of PSA and neuronal network activity alter synaptic plasticity, we examined the consequences of NEU inhibition on synaptic plasticity as a cellular basis for behavioral and cognitive functions. Using the specific ganglioside sialidase inhibitor 2deoxy-2,3-dehydro-N-acetylneuraminic acid (NADNA), we show that short-term blockade of endogenous NEU alters long-term synaptic potentiation (LTP) and frequency dependent plasticity in combined entorhinal cortex/hippocampal slices without an effect on paired-pulse facilitation and longterm depression (LTD). Application of low-frequency stimulation before the induction of LTP restores LTP in NADNApretreated slices to the control level. This data indicates that NADNA treatment induces potentiation of synaptic response, which results in the decrease in the magnitude of subsequent LTP.

\section{Methods}

All experimental procedures were performed in accordance with the guidelines set by the National Institutes of Health for the humane treatment of animals and approved by the Animal Care Committee of Bogomoletz Institute of Physiology.

2.1. Slice Preparation. Combined entorhinal cortex/hippocampal slices including neocortical areas (Te2 and Te3), entorhinal cortex, subiculum, and hippocampus were prepared from Wistar rats aged 19 to 21 days postnatally (P1921) as previously described with some modifications [21]. On the day of the experiment the rat was deeply anesthetized using isoflurane and decapitated. The brain was removed and placed into ice-cold oxygenated $\left(95 \% \mathrm{O}_{2}-5 \% \mathrm{CO}_{2}\right)$ artificial cerebrospinal fluid (ACSF) of the following composition (mM): $\mathrm{NaCl} 119, \mathrm{KCl} 2.5, \mathrm{CaCl}_{2} 2.0, \mathrm{MgSO}_{4} 1.3, \mathrm{NaHCO}_{3}$ 26, $\mathrm{NaH}_{2} \mathrm{PO}_{4}$ 1.2, and glucose 11 ( $\mathrm{pH}$ 7.35). Cerebellum, frontal lobe region (coronal section), and ventral-lateral areas (sections at the angle $20^{\circ}$ to $30^{\circ}$ off the horizontal axis) were removed from the brain. The remaining part of the brain was mounted on the stage of a Vibroslice NVSL (World Precision Instruments Inc., Sarasota, FL, USA) and cut $(400 \mu \mathrm{m})$ through the hemispheres at an angle of 30$35^{\circ}$ of their horizontal planes. For the experiments we took 3-4 slices from the dorsal part of the hippocampus [22]. Slices were maintained in an oxygenated ACSF at a room temperature for at least $1.5 \mathrm{~h}$ before use.
2.2. Electrophysiology. Brain slices were transferred to the incubation chamber and superfused with oxygenated ACSF at a rate of $2 \mathrm{~mL} / \mathrm{min}\left(22-24^{\circ} \mathrm{C}\right)$. Extracellular recordings were obtained within the CAl stratum radiatum (SR) of hippocampus with extracellular glass microelectrodes (3$4 \mathrm{M} \Omega$ ) filled with ACSF using patch-clamp amplifier (PC 501A, Warner Instruments Corp., Hamden, CT). Stimulating and recording electrodes were placed on the slice surface approximately $400 \mu \mathrm{m}$ apart from each other. Evoked postsynaptic responses were elicited by stimulation of Schaffer collateral-commissural pathway using a concentric bipolar stimulating electrode (FHC Inc., Bowdoin, ME) connected to a flexible stimulus isolator (ISO-Flex, A.M.P. Instruments, Jerusalem, Israel). Stimulation intensity varied between 150 and $400 \mu \mathrm{A}$ in all slices. At the beginning of each experiment maximal synaptic response was determined by generating input-output curves. For baseline recording the current intensity was set to elicit $30 \%$ of maximal response. The stimulation was applied every $30 \mathrm{~s}$. The stimulation protocol to induce synaptic plasticity was applied after 10-20 min of stable baseline recording. In paired-pulse experiments two stimuli were delivered to the hippocampal pathway with interstimulus intervals (ISI) ranging from 25 to $300 \mathrm{~ms}$. The paired-pulse ratio was defined as S2/S1, where S1 and S2 are slopes of the postsynaptic responses evoked by first and second pulses, respectively. To induce LTP either highfrequency tetanic stimulation (HFS) of 100 pulses at frequency of $100 \mathrm{~Hz}$ or a brief tetanic stimulation (15 pulses at $50 \mathrm{~Hz}$ ) was delivered at baseline stimulation intensity. Short-term depression (STD) was measured during $100 \mathrm{~Hz}$ and $50 \mathrm{~Hz}$ trains. To induce LTD low-frequency stimulation (LFS) of 1800 pulses at frequency of $1 \mathrm{~Hz}$ was delivered at baseline stimulation intensity. Recordings were digitized at $10 \mathrm{kHz}$ using an analogue-to-digital converter (National Instruments, Austin, TX) and stored on a computer using the WinWCP program (Strathclyde Electrophysiology Software, University of Strathclyde, Glasgow, UK).

2.3. NEU Blocker Treatment. Brain slices were incubated with NADNA during $2 \mathrm{hr}$ at room temperature then extensively washed with ACSF before recordings. In all experiments we used NADNA in concentration of $500 \mu \mathrm{M}$ purchased from Sigma-Aldrich (St. Louis, MO, USA). The specificity of the effect of NADNA as a blocker of the endogenous NEU was shown in histological and electrophysiological studies in our previous reports $[2,19]$.

2.4. Drugs. D-2-Amino-5-phosphonopentanoic acid (DAPV) was obtained from Tocris (Ellisville, MO, USA). All other chemicals were purchased from Sigma (St. Louis, MO, USA).

2.5. Data Analysis. Offline analysis of the recordings was performed using Clampfit (Axon Instruments, USA), Prism 5 (GraphPad, La Jolla, CA), and Origin 7.5 (OriginLab, Northampton, MA) software. Statistical comparison of the effects of NADNA treatment on different forms of plasticity 
(except STD during $100 \mathrm{~Hz}$ train) was performed by measuring the initial slope of the field response. The average field excitatory postsynaptic potential (fEPSP) slope during a 5 min period before LTP and LTD induction was taken as the baseline, and all values were normalized to this baseline. LTP magnitude was measured from 30 to $55 \mathrm{~min}$ after tetanic stimulation (during this time fEPSP was typically stable). In experiments where LFS preceded HFS, LTP magnitude was measured from 20 to $30 \mathrm{~min}$ after tetanic stimulation. The magnitude of fEPSP depression induced by LFS was estimated from 20 to $30 \mathrm{~min}$ after stimulation. During the above mentioned periods normalized fEPSP slopes were averaged for each slice and the mean values were then averaged and compared for control and NADNA-pretreated group. For measurement of STD during $50 \mathrm{~Hz}$ train all fEPSP slopes were normalized to fEPSP slope in response to the first pulse. The synaptic response to $100 \mathrm{~Hz}$ train stimulation was measured as a single entity and normalized to the prestimulus baseline fEPSP amplitude for each recording. The magnitude of STD was estimated during the last $100 \mathrm{~ms}$ of the train and averaged for each slice. Obtained mean values were then averaged for control and NADNA-pretreated groups and compared. Fiber volley (FV) amplitude was measured as a difference between the positive and the following negative peak. In experiments, where fEPSPs were blocked with glutamate receptor antagonist before HFS, FV amplitudes were normalized to the prestimulus baseline FV amplitude for each recording. Two-way repeated measures ANOVA and unpaired Student's $t$-test were used to analyze difference between groups. A $P$ value less than 0.05 was considered significant. Results were expressed as Mean \pm SEM; $n$ is the number of slices.

\section{Results}

Previously we showed that blockade of NEU activity leads to an increase in the density of simple and perforated synapses in hippocampal CA1 SR region [19]. To test whether newly formed synapses are functional, Shaffer collaterals were stimulated and field potential recordings were performed from the CA1 SR region in control and NADNA-pretreated slices (Figure 1(a)). To estimate the maximal field potential response in each recording the stimulation intensity was gradually increased until the amplitude of the response reached the saturation level. Input/output curves revealed a significant increase of the maximal rising slope of fEPSP in NADNA-pretreated slices compared to controls (NADNApretreated group: $0.20 \pm 0.05 \mathrm{mV} / \mathrm{ms}[n=21]$; control: $0.08 \pm$ $0.02 \mathrm{mV} / \mathrm{ms}[n=17], t_{36}=2.1, P<0.05$, Figure 1(b)(b2)) without alteration of FV amplitude (NADNA-pretreated group: $0.22 \pm 0.01 \mathrm{mV}[n=11]$; control: $0.20 \pm 0.01 \mathrm{mV}$ $[n=17], t_{19}=0.9, P=0.34$, Figure 1(b)(b1)). The coefficient of variation of the baseline fEPSP slope (30\% of the maximal response) was significantly decreased in the NADNA-pretreated group compared to controls: SD/Mean, $0.22 \pm 0.02[n=11]$ in control versus $0.10 \pm 0.04[n=9]$ after pretreatment with NADNA $\left(t_{18}=4.83, P<0.001\right.$, Figure 2). FEPSPs consist of N-methyl-D-aspartate (NMDA) and non-NMDA receptor-mediated components. To clarify whether NADNA pretreatment differently affects these components, we used specific NMDA receptor antagonist D-2-amino-5-phosphonopentanoic acid (D-APV) and nonNMDA receptor antagonist 6-cyano-7-nitroquinoxaline-2,3dione (CNQX). Application of $50 \mu \mathrm{M}$ D-APV produced a similar modest decrease in the baseline synaptic response in both groups $(91.1 \pm 3.5 \%[n=9]$ in control versus $85.8 \pm 5.0 \%[n=8]$ in NADNA-pretreated slices). The remaining component of fEPSP was completely attenuated by application of $10 \mu \mathrm{M}$ CNQX. These data suggest that NADNA pretreatment similarly affects NMDA and nonNMDA components of synaptic responses.

In the next set of experiments we investigated the effect of downregulation of NEU activity on the paired-pulse plasticity as a form of short-term synaptic plasticity reflecting changes in the release probability of presynaptic cell. Depending on the presynaptic release probability, repetitive stimulation could elicit either facilitation or depression [23]. Pairedpulse facilitation (PPF) of synaptic responses in NADNApretreated group was recorded in all slices irrespective of the duration of ISI. In control slices, paired-pulse depression of fEPSP was observed at ISI 25 and $50 \mathrm{~ms}$ in only two recordings out of twelve (16.7\%). At ISI 100 to $300 \mathrm{~ms}$ PPF of synaptic responses was observed in all control slices. Figure 3 indicates that regardless of the ISI the paired-pulse ratio (PPR) of postsynaptic responses did not change after NADNA pretreatment (control group $n=12$; NADNApretreated group $n=10 ; F_{1,80}=0.35, P=0.56$ ).

To study the effect of NEU inhibition on long-term synaptic plasticity we used a protocol for LTP induction consisting of 100 stimuli delivered at $100 \mathrm{~Hz}$. Figure 4(a) represents the group data of fEPSP evoked by stimulation of Shaffer collateral and recorded in CA1 SR region in the control and NADNA-pretreated groups before and after delivery of the HFS. An average maximal posttetanic potentiation was significantly decreased in the NADNA-pretreated group compared to controls $(254.2 \pm 28.3 \%[n=10]$ of baseline in the control group and $137.8 \pm 8.4 \%[n=9]$ of baseline in the NADNA-pretreated group, $t_{17}=3.76, P=0.002$ ). There was also a significant difference in LTP magnitude between NADNA-pretreated $(115.6 \pm 4.9 \%[n=9])$ and control $(155.0 \pm 13.2 \%[n=10])$ groups $\left(F_{1,850}=7.18, P=0.02\right)$.

During the high-frequency stimulus train fEPSP exhibited a strong depression. Figure 4(b) illustrates group data of the dynamic of evoked field potential changes during delivery of 100 pulses at $100 \mathrm{~Hz}$ in both groups. The relative field potential amplitudes at the end of the train (estimated during the last $100 \mathrm{~ms}$ of the train) were $37.2 \pm 0.4 \%[n=10]$ in control versus $8.8 \pm 0.1 \%[n=9]$ in NADNA-pretreated slices $\left(F_{1,153}=25.6, P<0.0001\right)$. Figure $4(\mathrm{c})$ shows that no difference in FV amplitude dynamic between control and NADNA-pretreated groups during HFS was observed in experiments where NMDA and non-NMDA receptor antagonists (50 $\mu \mathrm{M}$ D-APV and $10 \mu \mathrm{M}$ CNQX) were applied before HFS. These findings indicate that changes of STD level in the NADNA-pretreated group are due to the alteration of synaptic machinery. 


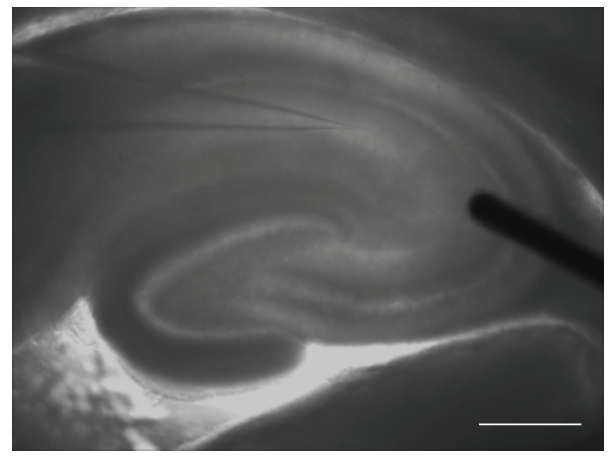

(a)

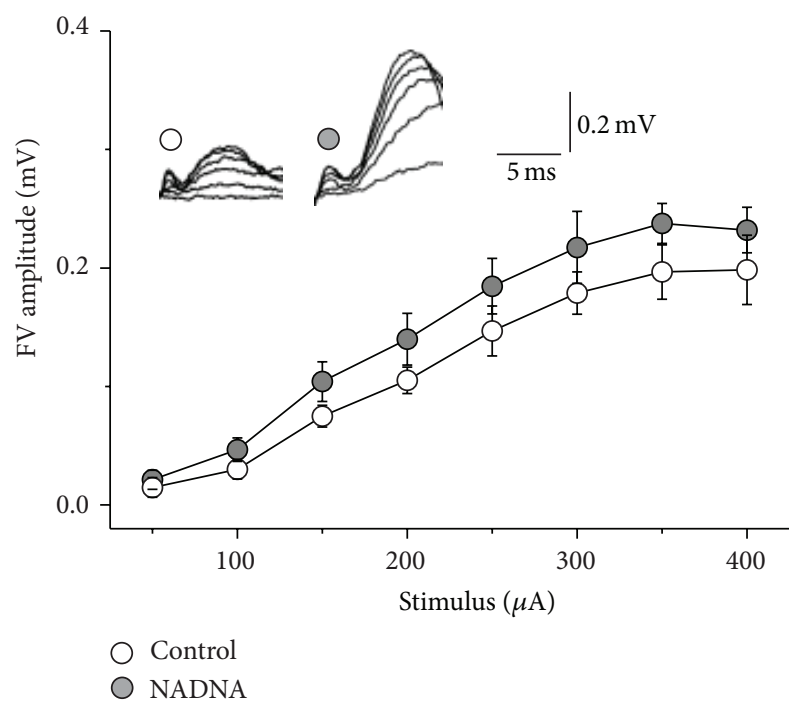

(b1)

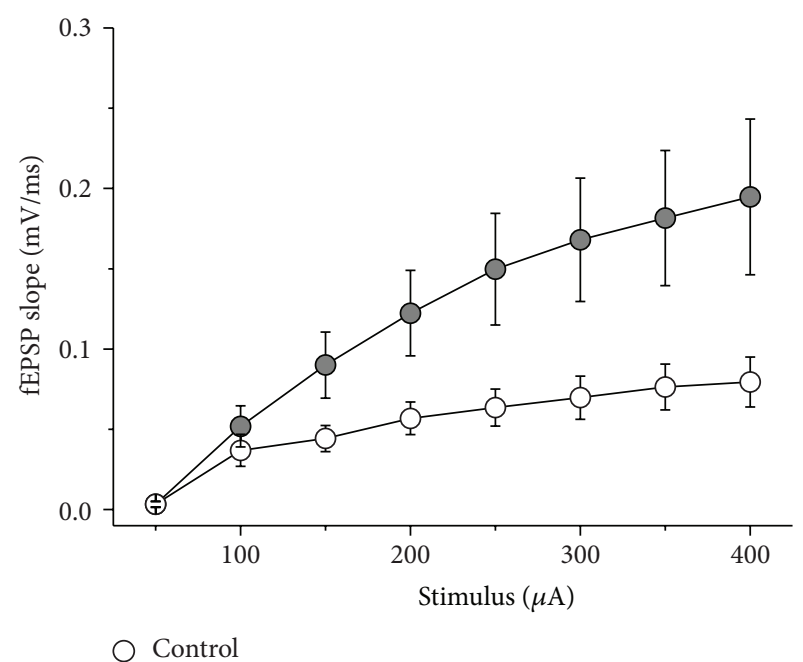

O NADNA

(b)

FIGURE 1: Inhibition of NEU activity affects basal evoked excitatory synaptic transmission. (a) The arrangement of electrodes for recordings. fEPSPs were evoked by stimulation of Shaffer collaterals using concentric electrode (right) and recorded using a glass electrode (left) filled with extracellular solution placed in CA1 SR region. Scale bar, $0.5 \mathrm{~mm}$. (b) Input-output curves show significant increase of the maximal fEPSP slope after suppression of NEU activity without the effect on the fiber volley (FV) amplitude.

To eliminate possible effects of depression during prolonged HFS on the posttetanic response, in the next set of experiments we used a brief tetanic stimulation protocol (15 pulses delivered at $50 \mathrm{~Hz}$ ) to induce synaptic potentiation [24]. In both groups repetitive stimuli evoked an initial facilitation of the fEPSP response followed by their gradual decrease with time. There was no significant difference in STD between groups (Figure 5(b), $F_{1,504}=0.02, P=0.88$ ). However, there was a significant difference in the maximal posttetanic potentiation between groups (controls: $179.7 \pm$ $6.7 \%$ [ $n=17]$ of baseline; NADNA-pretreated group: $154.6 \pm$ $4.5 \%[n=21]$ of baseline, $\left.t_{36}=3.2, P=0.003\right)$. Also, we found significant difference in the magnitude of LTP in the NADNA-pretreated group $(115.2 \pm 4.2 \%[n=21])$ compared to controls $\left(136.5 \pm 7.7 \%[n=17], F_{1,864}=6.58, P=0.01\right.$, Figure 5(a)).

Previous reports show that the downregulation of NEU activity and the increase in tissue sialylation enhance neuronal activity $[2,19,20,25,26]$. The increase in neuronal activity was shown to produce LTP-like changes [27], which can lead to the impairment of subsequent LTP induction. To examine the possibility that the decrease in LTP magnitude in NADNA-pretreated slices can be due to the saturation of LTP produced by increased neuronal activity we delivered LFS (1800 pulses at $1 \mathrm{~Hz}$ ) before applying the LTP protocol $(100$ pulses at $100 \mathrm{~Hz})$. LFS induces a similar reduction of fEPSP slope in both groups (LTD magnitude for control: $48.0 \pm 6.8 \%[n=19]$ of baseline and for NADNA-pretreated group: $58.0 \pm 12.9 \%\left[n=9\right.$ ] of baseline, $F_{1,234}=0.57, P=0.5$ ). After obtaining a new stable baseline (baseline 2; 20-30 min after LFS) high-frequency tetanic stimulation was applied. HFS induces the potentiation of postsynaptic responses in both groups. In these experiments we did not find statistically significant group differences in the LTP magnitude (control: $145.6 \pm 22.1 \%[n=19]$ of baseline 2 and NADNA-pretreated group $180.6 \pm 43.7 \%[n=9]$ of baseline $2, F_{1,234}=0.64$, $P=0.4$, Figure 6). 


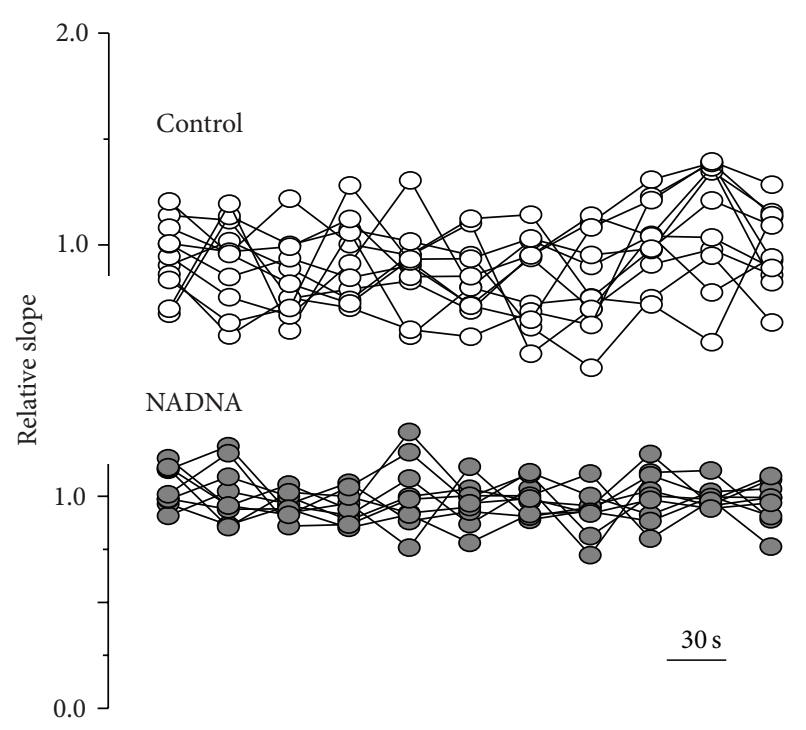

(a)

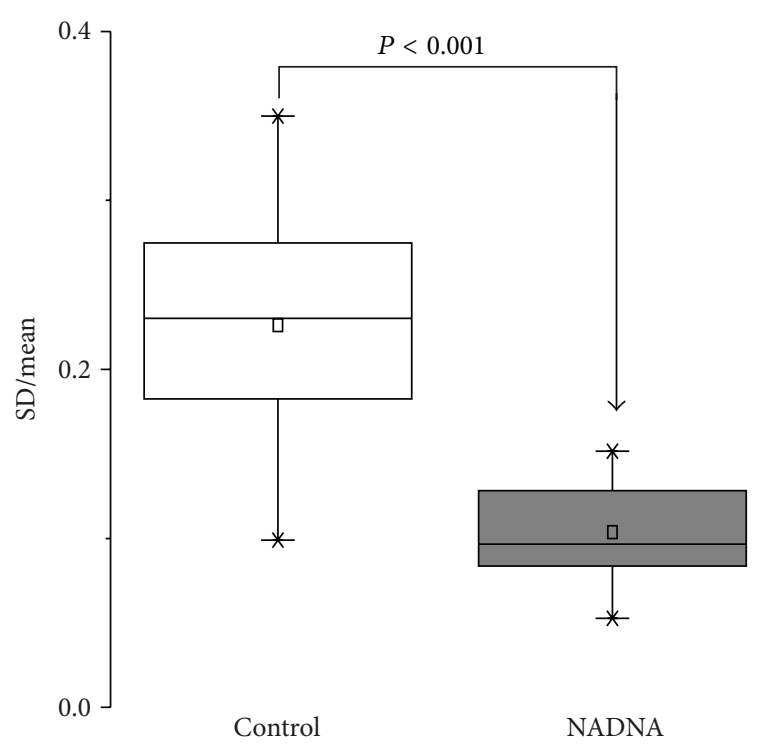

(b)

FIGURE 2: Effect of inhibition of NEU activity on variation of field potential response in hippocampal CA3-to-CA1 network. (a) Variation of baseline fEPSP recorded at $0.033 \mathrm{~Hz}$ in control and NADNA-pretreated slices. Each curve represents normalized to average baseline fEPSP slope from one slice. (b) Histogram shows that variation of baseline fEPSP slope significantly decreases in slices pretreated with NADNA.

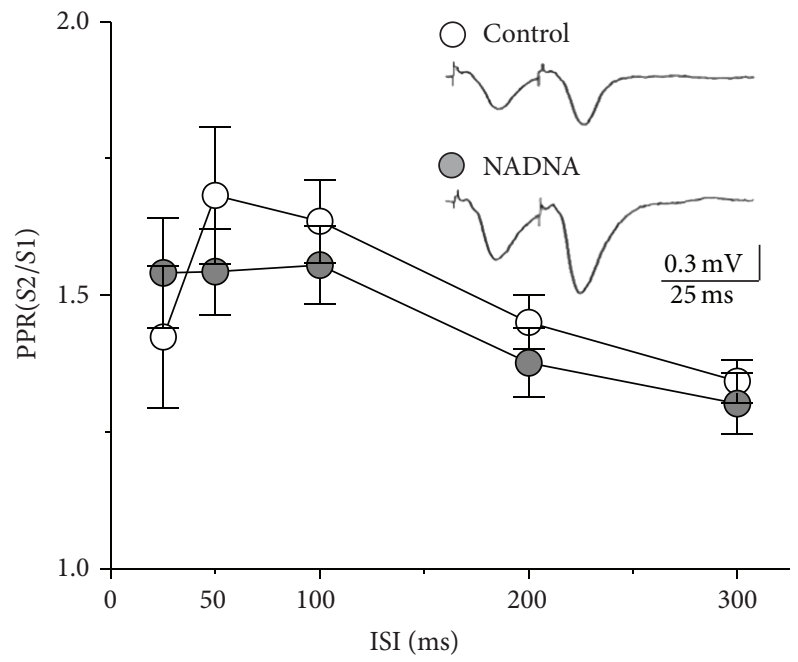

FIGURE 3: NEU inhibition does not influence paired-pulse plasticity in CA1 region of hippocampus. Histogram of average paired-pulse ratios as a function of ISI in control (white) and NADNA-pretreated (grey) slices. There were no significant differences between groups. Insets: averaged sample records of fEPSP at CA3-CA1 synapses measured at $25 \mathrm{~ms}$ ISI. All data are presented as Mean \pm SE.

\section{Discussion}

The present study demonstrates that suppression of endogenous NEU activity with the specific inhibitor NADNA (i) causes an increase in the field potential slope and a reduction in variation of fEPSP in CA3-to-CA1 network of rat hippocampus, (ii) does not alter paired-pulse plasticity and LTD, (iii) promotes STD during prolonged HFS, and (iv) decreases LTP magnitude; and (v) synaptic depotentiation restores the LTP deficit in NADNA-pretreated slices to the control levels. These data show that changes in the activity of endogenous
NEU can substantially modify hippocampal synaptic plasticity which may be the cellular correlate of behavioral and cognitive impairment associated with abnormal metabolism of NEU and altered PSA levels [13, 28, 29].

Significant increases in the proportion of perforated synapses in the CA1 SR region of hippocampus have been observed in cultured hippocampal slices pretreated with NADNA [19]. Alterations in the density of perforated synapses are considered as the morphological feature of activity-dependent synaptogenesis and have been reported under different physiological and pathological conditions 


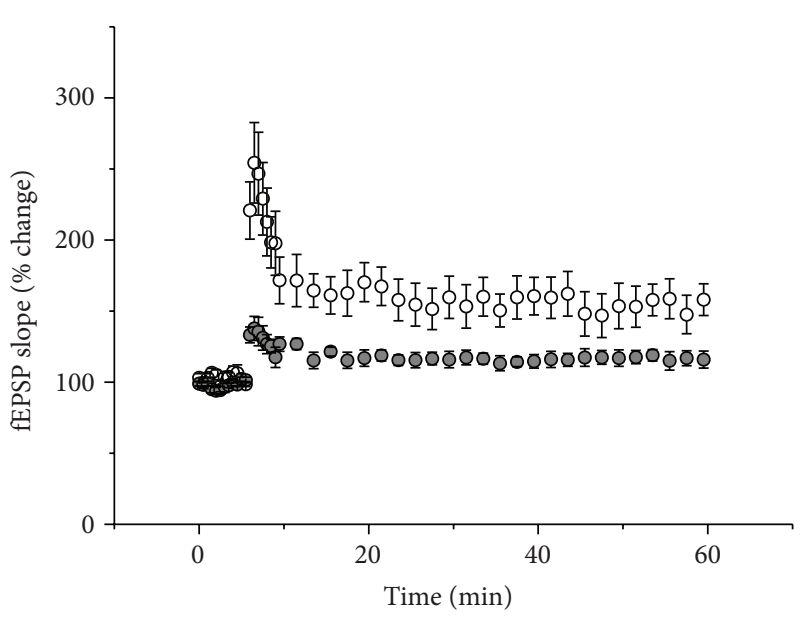

(a)

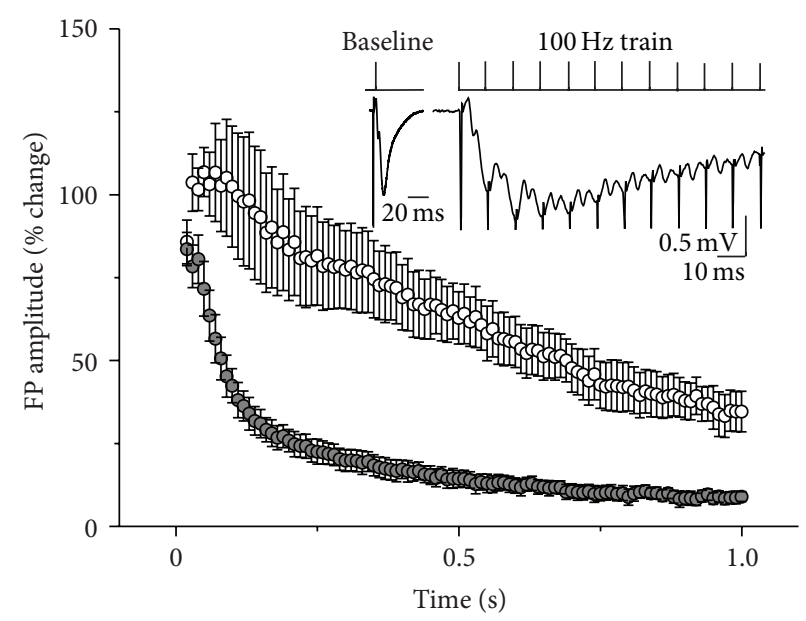

(b)

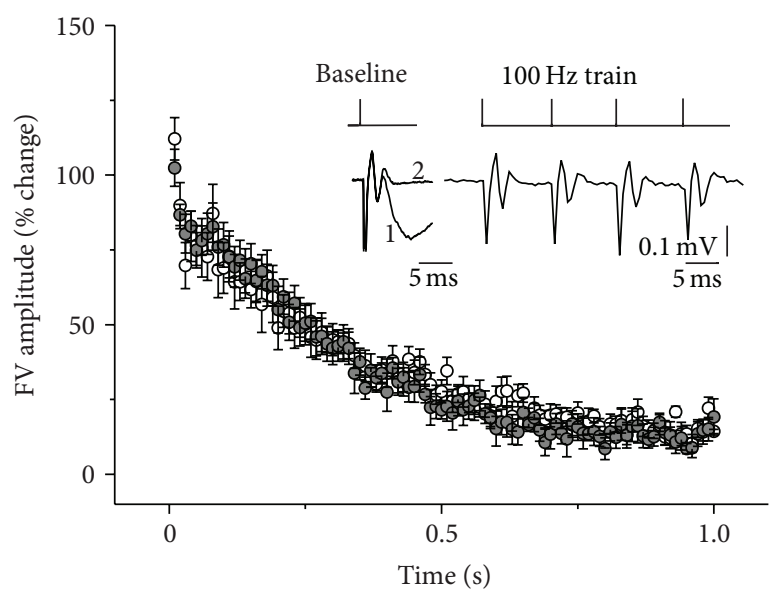

(c)

FIgURE 4: Effect of inhibition of NEU activity on the long-term potentiation and the short-term depression in hippocampal CA3-to-CA1 network induced by $100 \mathrm{~Hz}$ stimulus train. (a) Average of baseline-normalized initial slopes of fEPSP evoked by stimulation of Shaffer collateral before and after delivery of $100 \mathrm{~Hz}$ train in control (white) and NADNA-pretreated slices (grey). (b) Summarized changes in field potentials recorded during $100 \mathrm{~Hz}$ stimulation in control (white) and NADNA-pretreated slices (grey). Each point in the graph represents averaged amplitude of the field potential recorded at the time point of $9.5 \mathrm{~ms}$ after every stimulus delivery during train and normalized to baseline fEPSP amplitude. Inset: examples of baseline field potentials and fragment of field potential recording during $100 \mathrm{~Hz}$ stimulation from the same slice. (c) Summarized changes of fiber volley (FV) amplitude during $100 \mathrm{~Hz}$ stimulation in control (white) and NADNA-pretreated slices (grey) recorded in the presence of NMDA and non-NMDA receptor antagonists (resp., D-APV and CNQX). Each point in the graph represents averaged FV amplitude recorded after every stimulus delivery during the train and normalized to baseline FV amplitude. Inset: examples of baseline field potentials recorded from NADNA-pretreated slice before (1) and after (2) application of glutamate receptor blockers and fragment of field potential recording during $100 \mathrm{~Hz}$ stimulation from the same slice. All data are presented as Mean \pm SE.

[30-32]. In the present study we observed that NEU inhibition significantly increases postsynaptic responses while decreasing their variation. Such an effect is observed under condition of changes in neuronal excitability and following LTP induction [33]. Our data demonstrates that morphological changes caused by suppression of endogenous NEU activity parallel the enhanced functional excitatory synaptic connectivity in the hippocampal CA3-to-CA1 network. Additionally, direct PSA-AMPA receptor interactions were recently reported using exogenously applied bacterial PSA, colominic acid [34]. As AMPA receptor activity is the main contributor of fEPSP, we cannot exclude the possibility that increases in fEPSP may be partly due to direct potentiation of AMPA receptors through the increase in sialylation level caused by downregulation of NEU activity.

Inhibition of NEU activity did not affect paired-pulse plasticity. This data is in agreement with previous study where modulation of the PSA level by exogenously applied NEU did not affect short-term plasticity in CA3-to-CA1 networks of organotypic hippocampal culture [9]. In addition, pretreatment with NADNA did not affect LTD. Similar results were obtained previously using another NEU blocker, oseltamivir carboxylate, the active form of oseltamivir [35]. However, NADNA-pretreated slices show more pronounced depression 


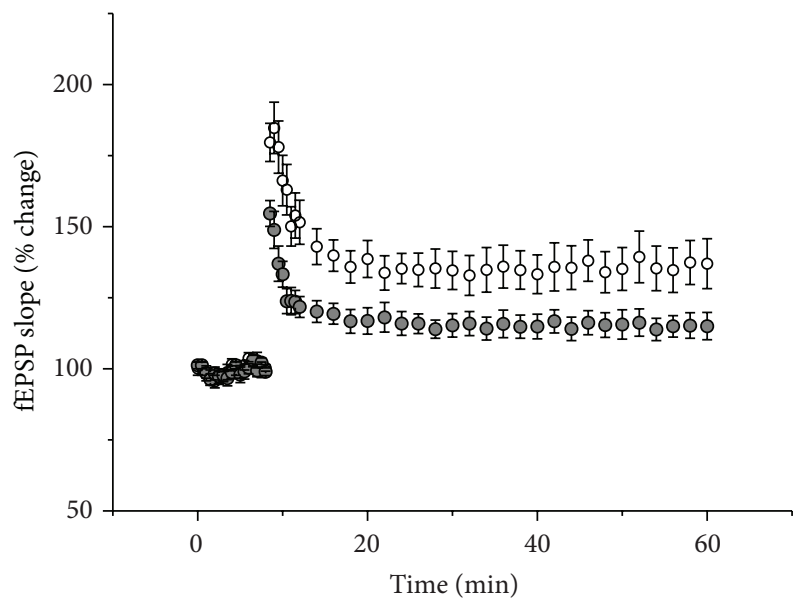

(a)

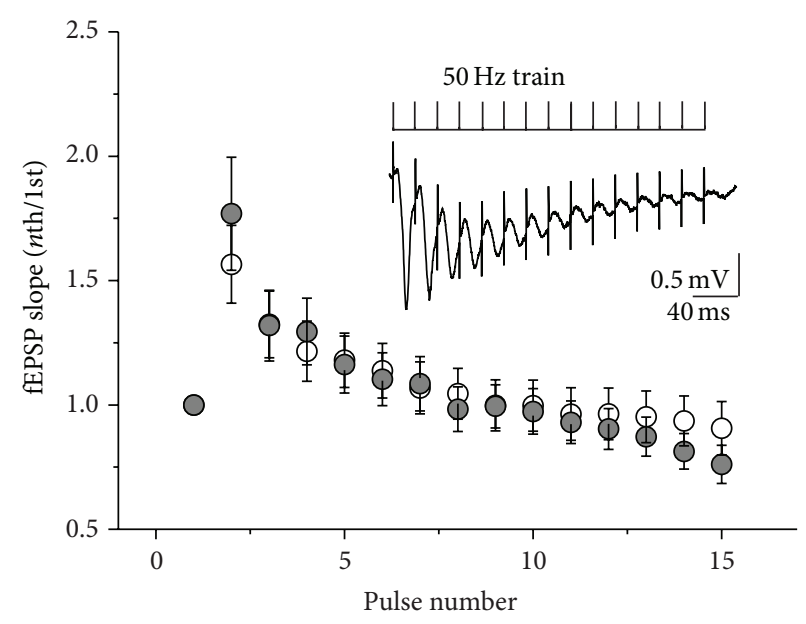

(b)

FIGURE 5: Effect of inhibition of NEU activity on the long-term potentiation and the short-term depression in hippocampal CA3-to-CA1 network induced by $50 \mathrm{~Hz}$ stimulus train. (a) Average of baseline-normalized initial slopes of fEPSP evoked by stimulation of Shaffer collateral before and after delivery of $50 \mathrm{~Hz}$ train in control (white) and NADNA-pretreated slices (grey). (b) Cumulative changes in field potentials recorded during $50 \mathrm{~Hz}$ stimulation in control (white) and NADNA-pretreated slices (grey). fEPSP slopes were normalized to the fEPSP slope in response to the first pulse and graphed versus pulse number. Inset: field potential recording during $50 \mathrm{~Hz} \mathrm{HFS}$.

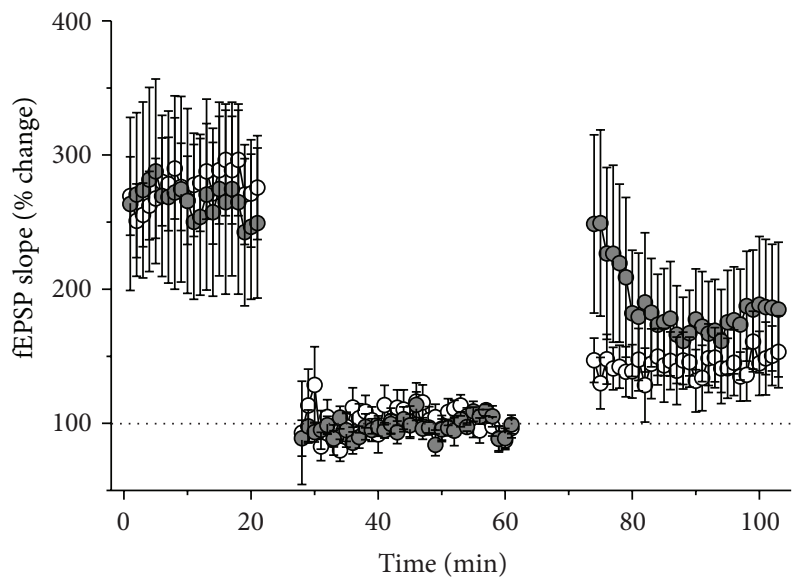

Figure 6: Synaptic depotentiation restores LTP in NADNA-pretreated slices to the control level. Pretreatment of slices with NADNA did not affect LTD of fEPSP evoked by LFS stimulation. Thirty min after the induction of LTD, tetanic stimulation of the Shaffer collaterals induces a similar potentiation of the field potential responses in NADNA-pretreated and control slices. All data are presented as Mean \pm SE.

in response to continuous HFS than control slices. Prolonged high-frequency stimulation leads to the transient decrease in synaptic strength due to vesicle depletion, inactivation of both release sites and calcium channels [36]. Another possible mechanism of depression during HFS is desensitization of postsynaptic receptors and activity-dependent receptor internalization [37, 38]. As NADNA pretreatment results in synaptic potentiation, both presynaptic and postsynaptic mechanism of STD during HFS could be altered in NADNApretreated slices.

In our study significant decrease in LTP magnitude was observed suggesting downregulation of synaptic effectiveness in conditions of NEU inhibition. Previous studies indicate that increase in membrane sialylation or downregulation of NEU activity could significantly alter neuronal activity. Increase of the sialylation level has been proposed to contribute to enhanced neuronal excitability after nerve injury [25]. Inhibition of endogenous NEU was reported to enhance neuronal synchronization in rat hippocampal CA3 region, increase the firing frequency and amplitude of spontaneous synchronous oscillations observed in CA1 region of cultured hippocampal slices, and intensify seizure-like activity in different in vitro and in vivo models of seizures $[2,19,20,26]$. It is well recognized that the ability of synaptic pathways to respond to stimulation with LTP is greatly dependent on the background activity as well as previous activation [39]. 
LTP can be more easily induced in conditions of decreased activity, while induction of LTP will be more difficult in synaptic pathways with a history of increased activity. Indeed, induction of LTP can be inhibited if weak stimulation is previously delivered to the same input pathway [40] or in $\mathrm{Mg}^{2+}$-free extracellular solution [41]. Also, learning is accompanied by reduced capability to induce LTP [42-44]. We hypothesize that NEU inhibition through modification of the neuronal activity produces LTP-like changes in the CA1 region of hippocampus, which results in a decrease in magnitude of subsequent LTP. Our experiments, where synaptic depotentiation completely restores LTP deficit in NADNA-pretreated slices to the control level, support this assumption.

Many studies show substantial contributions of PSA in plasticity and memory processing in both the developing and mature brain [29]. The peak of the expression of PSA is correlated with the critical period of neuronal development and PSA has been shown to play an important role in developmental structural and synaptic plasticity. In the adult brain, substantial PSA expression was found in restricted brain regions of continuous neurogenesis including hippocampus, amygdala, and neocortex. These areas have been shown to play an important role in different aspects of memory [10]. Decreases in the level of membrane sialylation impair spatial memory and suppress LTP in hippocampal slices $[6,9,12]$. Impairment of learning and long-term plasticity in CA3-CA1 synapses was demonstrated in mice lacking the polysialyltransferase, an enzyme essential for PSA biosynthesis [10]. On the other hand, exogenous application of PSA impairs LTP and formation of hippocampus-dependent contextual memory [45]. Elevated PSA levels have been associated with various neuropsychiatric disorders such as temporal lobe epilepsy, Alzheimer disease, and chronic stress [46-48]. Short-term inhibition of NEU activity results in an increase of PSA level and neuronal excitability and leads to activitydependent synaptogenesis in CA1 region of hippocampus $[2,19,26]$. We suggest that impairment of LTP shown in the present study is a consequence of these alterations. These findings indicate that rigorous regulation of the PSA level (regardless of the direction) is a necessary component for normal LTP induction.

\section{Conclusions}

The present study emphasizes the importance of proper functioning of endogenous NEU in neuronal tissue. Our results support the idea that impairment of NEU activity affects hippocampal plasticity and provides insight into the cellular mechanisms underlying inherited disorders of impaired metabolism of NEU and neuropsychiatric disorders followed by changes in membrane sialylation and NEU activity. Moreover, considering that NEU inhibitor derivatives are widely used as anti-influenza drugs and have been reported to cause serious psychiatric side effects in patients receiving these drugs, our data can be used to better understand a possible mechanism of adverse effects of these antiviral drugs and hopefully improve their quality $[28,49]$.

\section{Conflict of Interests}

The authors declare that there is no conflict of interests regarding the publication of this paper.

\section{Acknowledgments}

This work was supported by the State Foundation of Fundamental Research of Ukraine F46.2/001, State Agency on Science, Innovations and Information of Ukraine and NIH NS073083, NS074450, and the Emmory R. Shapses Research Fund and Michael J. Pietroniro Research Fund.

\section{References}

[1] G. Rougon, "Structure, metabolism and cell biology of polysialic acids," European Journal of Cell Biology, vol. 61, no. 2, pp. 197207, 1993.

[2] D. Isaev, E. Isaeva, T. Shatskih et al., "Role of extracellular sialic acid in regulation of neuronal and network excitability in the rat hippocampus," Journal of Neuroscience, vol. 27, no. 43, pp. 11587-11594, 2007.

[3] L. Bonfanti and D. T. Theodosis, "Polysialic acid and activitydependent synapse remodeling," Cell Adhesion and Migration, vol. 3, no. 1, pp. 43-50, 2009.

[4] A. R. Ednie and E. S. Bennett, "Modulation of voltage-gated ion channels by sialylation," Comprehensive Physiology, vol. 2, no. 2, pp. 1269-1301, 2012.

[5] U. Rutishauser and L. Landmesser, "Polysialic acid in the vertebrate nervous system: a promoter of plasticity in cell-cell interactions," Trends in Neurosciences, vol. 19, no. 10, pp. 422427, 1996.

[6] C. G. Becker, A. Artola, R. Gerardy-Schahn, T. Becker, H. Welzl, and M. Schachner, "The polysialic acid modification of the neural cell adhesion molecule is involved in spatial learning and hippocampal long-term potentiation," Journal of Neuroscience Research, vol. 45, no. 2, pp. 143-152, 1996.

[7] E. Bennett, M. S. Urcan, S. S. Tinkle, A. G. Koszowski, and S. R. Levinson, "Contribution of sialic acid to the voltage dependence of sodium channel gating: a possible electrostatic mechanism," Journal of General Physiology, vol. 109, no. 3, pp. 327-343, 1997.

[8] D. Isaev, Q. Zhao, J. K. Kleen et al., "Neuroaminidase reduces interictal spikes in a rat temporal lobe epilepsy model," Epilepsia, vol. 52, no. 3, pp. e12-e15, 2011.

[9] D. Muller, C. Wang, G. Skibo et al., "PSA-NCAM is required for activity-induced synaptic plasticity," Neuron, vol. 17, no. 3, pp. 413-422, 1996.

[10] L. Bonfanti, "PSA-NCAM in mammalian structural plasticity and neurogenesis," Progress in Neurobiology, vol. 80, no. 3, pp. 129-164, 2006.

[11] E. Isaeva, I. Lushnikova, A. Savrasova, G. Skibo, G. L. Holmes, and D. Isaev, "Effect of neuraminidase treatment on persistent epileptiform activity in the rat hippocampus," Pharmacological Reports, vol. 63, no. 3, pp. 840-844, 2011.

[12] C. Venero, A. I. Herrero, K. Touyarot et al., "Hippocampal upregulation of NCAM expression and polysialylation plays a key role on spatial memory," European Journal of Neuroscience, vol. 23, no. 6, pp. 1585-1595, 2006.

[13] N. de Geest, E. Bonten, L. Mann, J. de Sousa-Hitzler, C. Hahn, and A. d'Azzo, "Systemic and neurologic abnormalities distinguish the lysosomal disorders sialidosis and galactosialidosis in 
mice," Human Molecular Genetics, vol. 11, no. 12, pp. 1455-1464, 2002.

[14] J. R. Buchhalter, "Inherited epilepsies of childhood," Journal of Child Neurology, vol. 9, supplement 1, pp. S12-S19, 1994.

[15] P. Wielgat, A. Walesiuk, and J. J. Braszko, "Effects of chronic stress and corticosterone on sialidase activity in the rat hippocampus," Behavioural Brain Research, vol. 222, no. 2, pp. 363367, 2011.

[16] A. Boyzo, J. Ayala, R. Gutiérrez, and J. Hernández-R, "Neuraminidase activity in different regions of the seizing epileptic and non-epileptic brain," Brain Research, vol. 964, no. 2, pp. 211217, 2003.

[17] E. A. Hale, S. K. Raza, R. G. Ciecierski, and P. Ghosh, "Deleterious actions of chronic ethanol treatment on the glycosylation of rat brain clusterin," Brain Research, vol. 785, no. 1, pp. 158-166, 1998.

[18] M. A. Azuine, S. J. Patel, and M. R. Lakshman, "Effects of chronic ethanol administration on the activities and relative synthetic rates of myelin and synaptosomal plasma membraneassociated sialidase in the rat brain," Neurochemistry International, vol. 48, no. 1, pp. 67-74, 2006.

[19] E. Isaeva, I. Lushnikova, A. Savrasova, G. Skibo, G. L. Holmes, and D. Isaev, "Blockade of endogenous neuraminidase leads to an increase of neuronal excitability and activity-dependent synaptogenesis in the rat hippocampus," European Journal of Neuroscience, vol. 32, no. 11, pp. 1889-1896, 2010.

[20] A. Usami, T. Sasaki, N. Satoh et al., "Oseltamivir enhances hippocampal network synchronization," Journal of Pharmacological Sciences, vol. 106, no. 4, pp. 659-662, 2008.

[21] D. Isaev, G. Ivanchick, V. Khmyz et al., "Surface charge impact in low-magnesium model of seizure in rat hippocampus," Journal of Neurophysiology, vol. 107, no. 1, pp. 417-423, 2012.

[22] C. Papatheodoropoulos and G. Kostopoulos, "Decreased ability of rat temporal hippocampal CA1 region to produce long-term potentiation," Neuroscience Letters, vol. 279, no. 3, pp. 177-180, 2000.

[23] R. S. Zucker and W. G. Regehr, "Short-term synaptic plasticity," Annual Review of Physiology, vol. 64, pp. 355-405, 2002.

[24] E. Kim, B. Owen, W. R. Holmes, and L. M. Grover, "Decreased afferent excitability contributes to synaptic depression during high-frequency stimulation in hippocampal area CA1," Journal of Neurophysiology, vol. 108, no. 7, pp. 1965-1976, 2012.

[25] X.-Q. Peng, X.-L. Zhang, Y. Fang, W.-R. Xie, and Y.-K. Xie, "Sialic acid contributes to hyperexcitability of dorsal root ganglion neurons in rats with peripheral nerve injury," Brain Research, vol. 1026, no. 2, pp. 185-193, 2004.

[26] A. V. Savrasova, I. V. Lushnikova, E. V. Isaeva, G. G. Skibo, D. S. Isaev, and P. G. Kostyuk, "The effect of neuraminidase blocker on gabazine-induced seizures in rat hippocampus," Fiziolohichny̌ Zhurnal, vol. 56, no. 4, pp. 14-18, 2010.

[27] S. L. Morgan and T. J. Teyler, "Epileptic-like activity induces multiple forms of plasticity in hippocampal area CA1," Brain Research, vol. 917, no. 1, pp. 90-96, 2001.

[28] M. Suzuki and Y. Masuda, "Effect of a neuraminidase inhibitor (oseltamivir) on mouse jump-down behavior via stimulation of dopamine receptors," Biomedical Research, vol. 29, no. 5, pp. 233-238, 2008.

[29] U. Rutishauser, "Polysialic acid in the plasticity of the developing and adult vertebrate nervous system," Nature Reviews Neuroscience, vol. 9, no. 1, pp. 26-35, 2008.
[30] D. G. Jones and R. K. S. Calverley, "Frequency of occurrence of perforated synapses in developing rat neocortex," Neuroscience Letters, vol. 129, no. 2, pp. 189-192, 1991.

[31] H. N. Noristani, R. S. Meadows, M. Olabarria, A. Verkhratsky, and J. J. Rodríguez, "Increased hippocampal CA1 density of serotonergic terminals in a triple transgenic mouse model of Alzheimer's disease: an ultrastructural study," Cell Death and Disease, vol. 2, no. 9, article e210, 2011.

[32] Y. W. Ruan, X. J. Han, Z. S. Shi, Z. G. Lei, and Z. C. Xu, "Remodeling of synapses in the CA1 area of the hippocampus after transient global ischemia," Neuroscience, vol. 218, pp. 268$277,2012$.

[33] G. A. Kerchner and R. A. Nicoll, "Silent synapses and the emergence of a postsynaptic mechanism for LTP," Nature Reviews Neuroscience, vol. 9, no. 11, pp. 813-825, 2008.

[34] T. Vaithianathan, K. Matthias, B. Bahr et al., "Neural cell adhesion molecule-associated polysialic acid potentiates $\alpha$ amino-3-hydroxy-5-methylisoxazole-4-propionic acid receptor currents," The Journal of Biological Chemistry, vol. 279, no. 46, pp. 47975-47984, 2004.

[35] Y. Izumi, K. Tokuda, K. A. O’Dell, C. F. Zorumski, and T. Narahashi, "Synaptic and behavioral interactions of oseltamivir (Tamiflu) with neurostimulants," Human and Experimental Toxicology, vol. 27, no. 12, pp. 911-917, 2008.

[36] D. Fioravante and W. G. Regehr, "Short-term forms of presynaptic plasticity," Current Opinion in Neurobiology, vol. 21, no. 2, pp. 269-274, 2011.

[37] A. C. Gambrill, G. P. Storey, and A. Barria, "Dynamic regulation of NMDA receptor transmission," Journal of Neurophysiology, vol. 105, no. 1, pp. 162-171, 2011.

[38] M. Heine, L. Groc, R. Frischknecht et al., "Surface mobility of postsynaptic AMPARs tunes synaptic transmission," Science, vol. 320, no. 5873, pp. 201-205, 2008.

[39] E. L. Bienenstock, L. N. Cooper, and P. W. Munro, “Theory for the development of neuron selectivity: orientation specificity and binocular interaction in visual cortex," Journal of Neuroscience, vol. 2, no. 1, pp. 32-48, 1982.

[40] Y.-Y. Huang, A. Colino, D. K. Selig, and R. C. Malenka, “The influence of prior synaptic activity on the induction of longterm potentiation," Science, vol. 255, no. 5045, pp. 730-733, 1992.

[41] E. J. Coan, A. J. Irving, and G. L. Collingridge, "Low-frequency activation of the NMDA receptor system can prevent the induction of LTP," Neuroscience Letters, vol.105, no. 1-2, pp. 205210, 1989.

[42] A. Kirkwood, M. G. Rioult, and M. F. Bear, "Experiencedependent modification of synaptic plasticity in visual cortex," Nature, vol. 381, no. 6582, pp. 526-528, 1996.

[43] D. Lebel, Y. Grossman, and E. Barkai, "Olfactory learning modifies predisposition for long-term potentiation and long-term depression induction in the rat piriform (olfactory) cortex," Cerebral Cortex, vol. 11, no. 6, pp. 485-489, 2001.

[44] M.-S. Rioult-Pedotti, D. Friedman, G. Hess, and J. P. Donoghue, "Strengthening of horizontal cortical connections following skill learning," Nature Neuroscience, vol. 1, no. 3, pp. 230-234, 1998.

[45] O. Senkov, M. Sun, B. Weinhold, R. Gerardy-Schahn, M. Schachner, and A. Dityatev, "Polysialylated neural cell adhesion molecule is involved in induction of long-term potentiation and memory acquisition and consolidation in a fear-conditioning paradigm," Journal of Neuroscience, vol. 26, no. 42, pp. 10888109898, 2006. 
[46] K. Pham, J. Nacher, P. R. Hof, and B. S. McEwen, "Repeated restraint stress suppresses neurogenesis and induces biphasic PSA-NCAM expression in the adult rat dentate gyrus," European Journal of Neuroscience, vol. 17, no. 4, pp. 879-886, 2003.

[47] R. Miettinen, M. Soininen, H. Tapiola, T. Alafuzoff, and I. Miettinen, "Hippocampal plasticity in Alzheimer's disease: changes in highly polysialylated NCAM immunoreactivity in the hippocampal formation," European Journal of Neuroscience, vol. 11, no. 5, pp. 1754-1764, 1999.

[48] M. Mikkonen, H. Soininen, R. Kälviäinen et al., "Remodeling of neuronal circuitries in human temporal lobe epilepsy: increased expression of highly polysialylated neural cell adhesion molecule in the hippocampus and the entorhinal cortex," Annals of Neurology, vol. 44, no. 6, pp. 923-934, 1998.

[49] Y. Izumi, K. Tokuda, K. A. O’Dell, C. F. Zorumski, and T. Narahashi, "Neuroexcitatory actions of Tamiflu and its carboxylate metabolite," Neuroscience Letters, vol. 426, no. 1, pp. 54-58, 2007. 

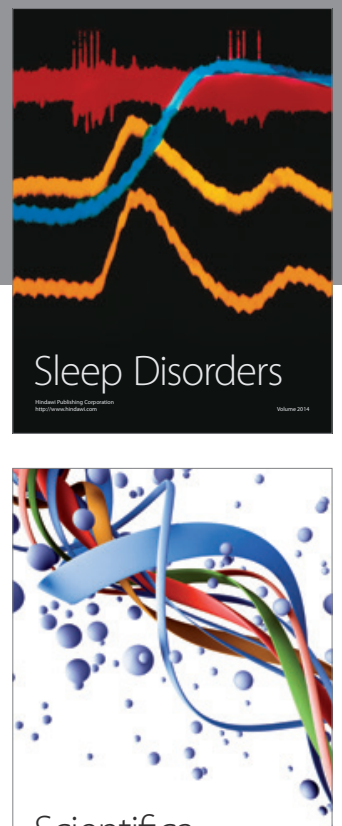

Scientifica
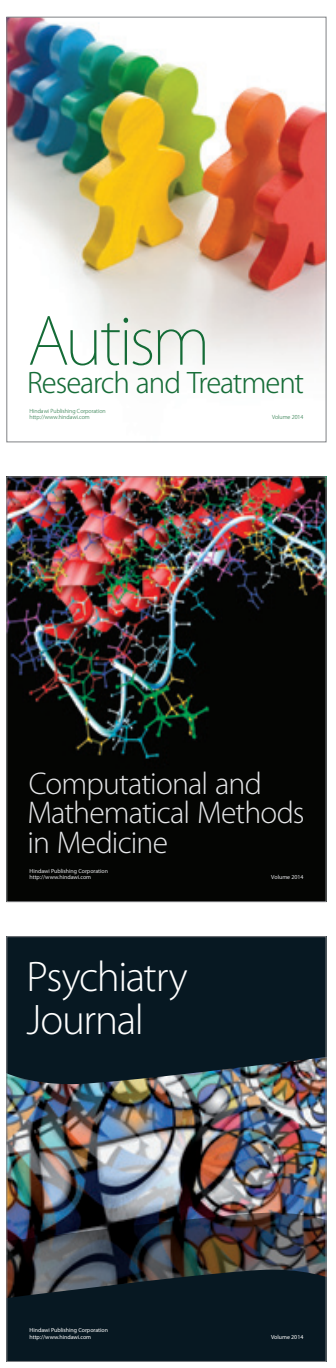
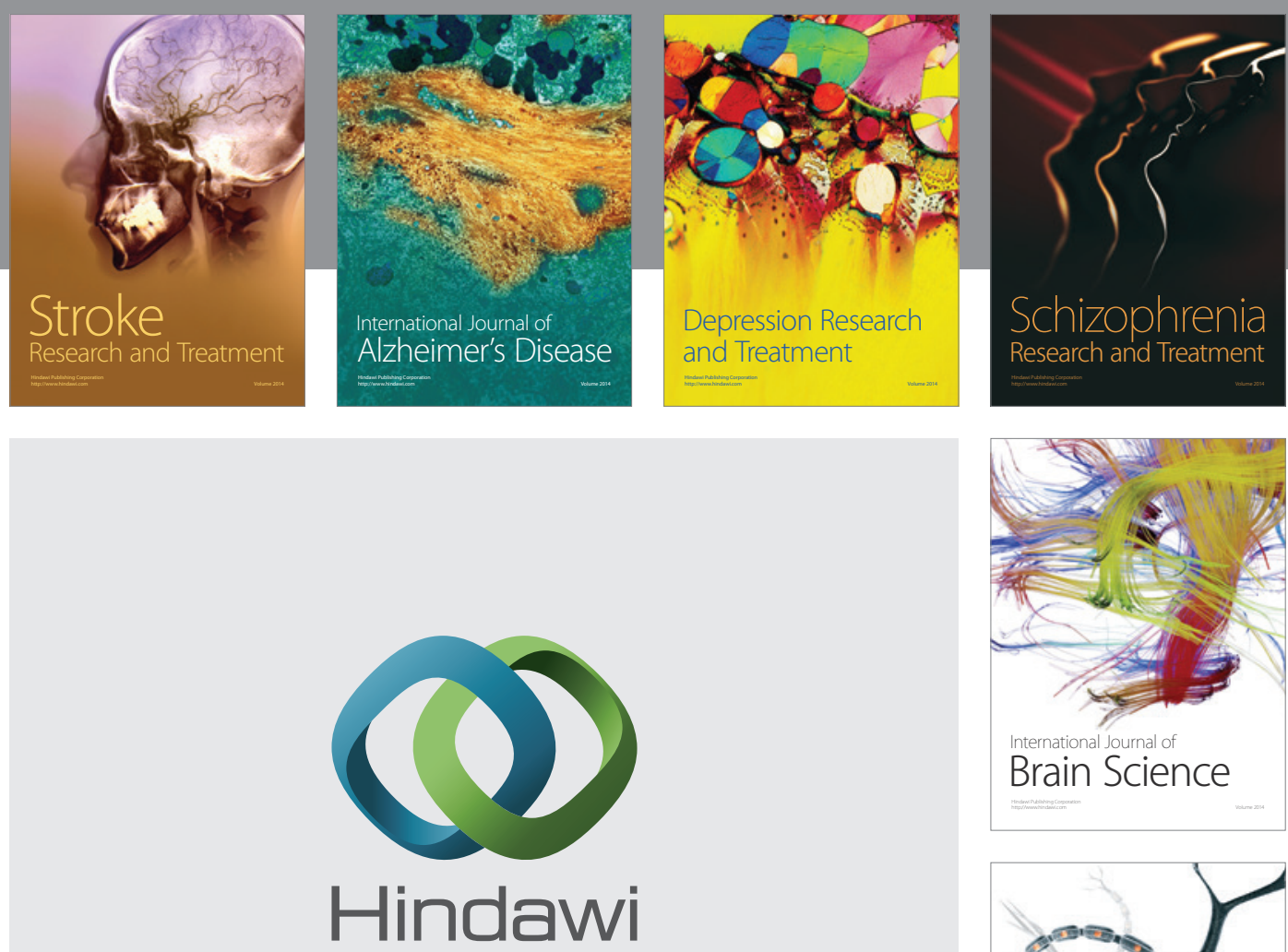

Submit your manuscripts at

http://www.hindawi.com
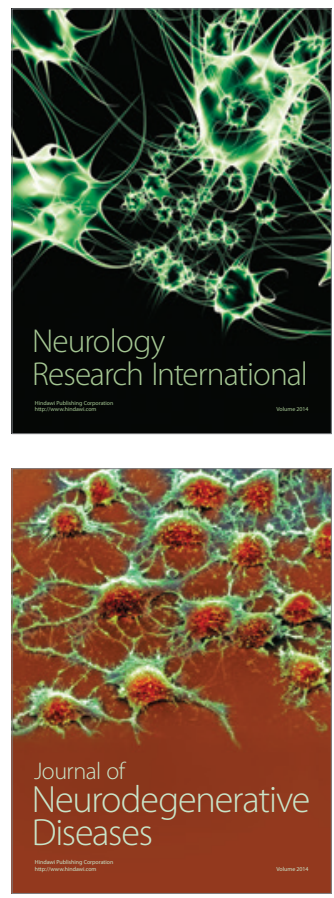

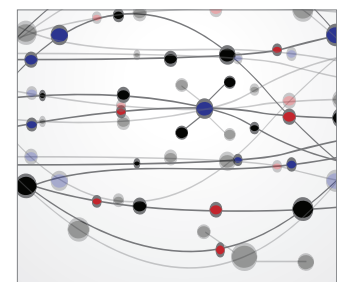

The Scientific World Journal
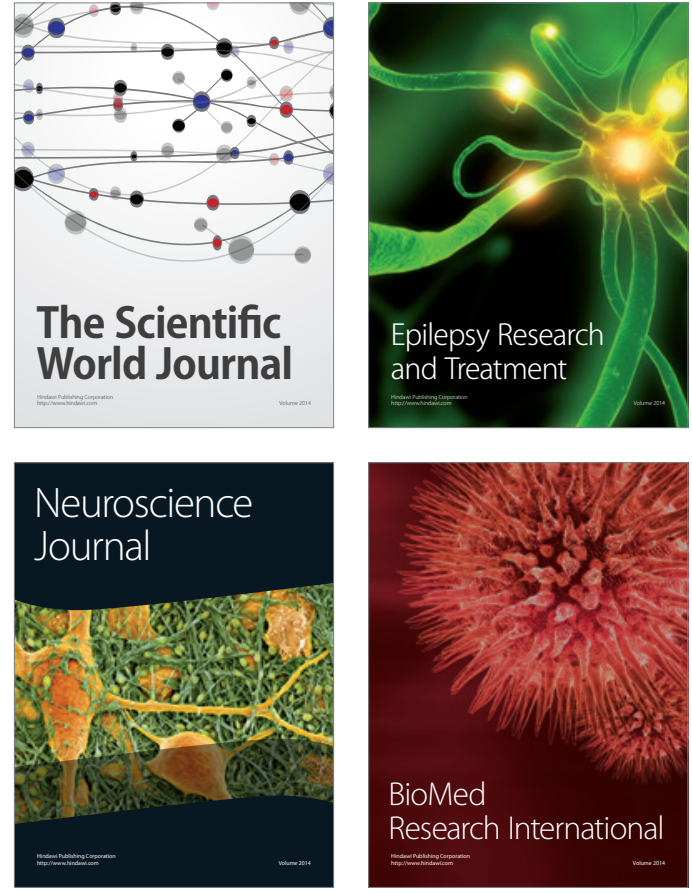

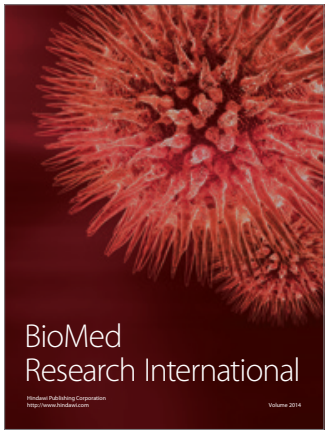

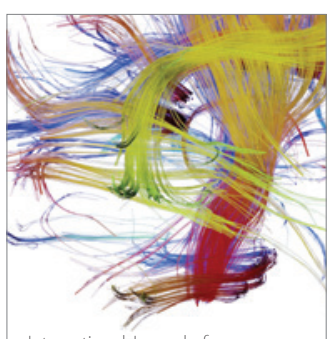

Brain Science

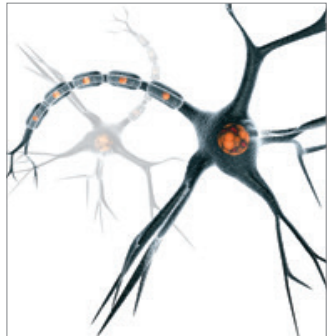

Neural Plasticity
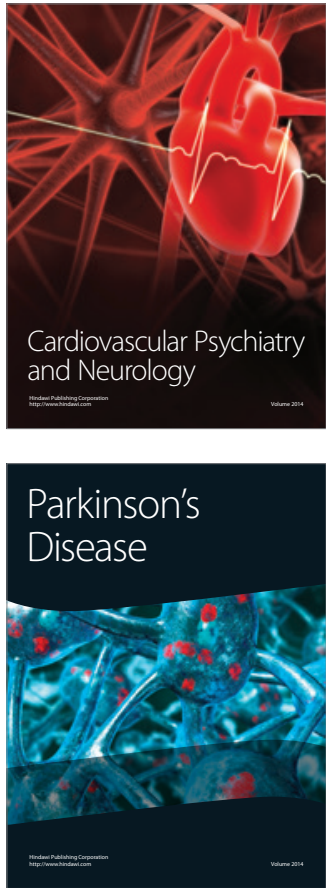